\title{
Frequency Adaptation for Enhanced Radiation Force Amplitude in Dynamic Elastography
}

\author{
Abderrahmane Ouared, Emmanuel Montagnon, Siavash Kazemirad, Louis Gaboury, \\ André Robidoux, and Guy Cloutier, Senior Member, IEEE
}

\begin{abstract}
In remote dynamic elastography, the amplitude of the generated displacement field is directly related to the amplitude of the radiation force. Therefore, displacement improvement for better tissue characterization requires the optimization of the radiation force amplitude by increasing the push duration and/or the excitation amplitude applied on the transducer. The main problem of these approaches is that the Food and Drug Administration (FDA) thresholds for medical applications and transducer limitations may be easily exceeded. In the present study, the effect of the frequency used for the generation of the radiation force on the amplitude of the displacement field was investigated. We found that amplitudes of displacements generated by adapted radiation force sequences were greater than those generated by standard nonadapted ones (i.e., single push acoustic radiation force impulse and supersonic shear imaging). Gains in magnitude were between 20 to $158 \%$ for in vitro measurements on agar-gelatin phantoms, and 170 to $336 \%$ for ex vivo measurements on a human breast sample, depending on focus depths and attenuations of tested samples. The signal-to-noise ratio was also improved more than 4 -fold with adapted sequences. We conclude that frequency adaptation is a complementary technique that is efficient for the optimization of displacement amplitudes. This technique can be used safely to optimize the deposited local acoustic energy without increasing the risk of damaging tissues and transducer elements.
\end{abstract}

\section{INTRODUCTION}

$\mathrm{O}$ VER the last two decades, the acoustic radiation force strategy has been increasingly used in remote dy-

Manuscript received February 20, 2015; accepted May 7, 2015. This work was supported by the Natural Sciences and Engineering Research Council of Canada (CHRP-365656-09) and by the Canadian Institutes of Health Research (CPG-95288). It is now supported by the Fonds Québécois de la Recherche sur la Nature et les Technologies (FQRNTPR-174387).

A. Ouared, S. Kazemirad, and G. Cloutier are with and E. Montagnon was with the Laboratory of Biorheology and Medical Ultrasonics, University of Montréal Hospital Research Center (CRCHUM), Montréal, QC H2X OA9, Canada.

A. Ouared and G. Cloutier are with the Institute of Biomedical Engineering, University of Montréal, Montréal, QC H3C 3J7, Canada.

E. Montagnon is with Rheolution Inc., Montréal, QC H2S 2X3, Canada.

L. Gaboury is with the Department of Pathology, University of Montréal Hospital, Montréal, QC H2W 1T7, Canada, and the Institute of Research in Immunology and Cancerology of the University of Montréal, Montréal, QC H3C 3J7, Canada.

A. Robidoux is with the Breast Cancer Research Group, University of Montréal Hospital Research Center, Montréal, QC H2W 1T7, Canada, and the Department of Surgery, University of Montréal, Montréal, QC H3C 3J7, Canada.

G. Cloutier is also with the Department of Radiology, Radio-Oncology and Nuclear Medicine, University of Montréal, Montréal, QC H3C 3J7, Canada (e-mail: guy.cloutier@umontreal.ca).

DOI http://dx.doi.org/10.1109/TUFFC.2015.007023 namic elastography for tissue characterization [1]-[4]. Dynamic elastography is now widely used and has been applied to study different organs or structures, such as the breast [5]-[7], liver [8]-[13], tendon [14], kidney [15], heart [16], [17], and venous thrombosis [18], to name a few examples. Elastic properties of tissues can be estimated by different methods with this approach. For instance, in [5], [19] mechanical properties of probed media were obtained from the velocity of shear waves estimated using the time of flight algorithm. The shear wave velocity is directly linked to the elasticity of the medium through $E \approx 3 \mu$ and $\mu=\rho v_{\mathrm{s}}^{2}$ (where $E=$ Young's modulus, $\mu=$ shear modulus, $\rho=$ density, and $v_{s}=$ the velocity of propagating shear waves). In more recent studies [8], [9], the shear wave velocity was estimated at different frequencies and the elasticity and viscosity components were calculated by fitting the experimental data to a Voigt rheological model. In other techniques [20], [21], viscoelastic parameters were obtained through analytical and/or numerical solutions of inverse problems.

The main clinically used remote dynamic elastography techniques are acoustic radiation force impulse (ARFI) [22] and supersonic shear imaging (SSI) [4]. In ARFI, one focused acoustic radiation force push is generated, and then the focus is moved axially and the beam laterally by adjusting delays for exciting transducer elements to scan the whole area. In the case of SSI, a quasi-planar shear wave front is generated by rapidly moving the focused push location axially. These remote palpation techniques are implemented on commercial scanners but suffer from strong attenuation of the shear wave amplitude with propagation on a few millimeters, which affects the signalto-noise ratio (SNR) and the biological tissue characterization accuracy, especially at high shear wave frequency [23].

The amplitude of the shear wave induced displacements is directly linked to the deposited radiation force intensity. To optimize the displacement amplitude, increasing the push duration and excitation voltage transmitted to the transducer were proposed [24], [25]. However, energy thresholds imposed by the Food and Drug Administration (FDA) can be easily exceeded, and consequently the biological tissue and the ultrasound (US) transducer may be damaged. The aim of the present study was to investigate the effect of the frequency used for the generation of the radiation force on the displacement field amplitude and on the SNR. The radiation pressure intensity depends on the acoustic wave frequency and distance from the US probe 
(i.e., focus depth). A theoretical model was developed to predict the optimal frequency depending on the attenuation of the medium and the focus depth. We show that the amplitude of displacements generated by a radiation force with an adapted frequency scheme is 20 to $336 \%$ greater than that generated at the central frequency of the transducer. The technique was applied in vitro on several homogeneous and heterogeneous agar-gelatin phantoms, and ex vivo on a human breast sample obtained after a complete mastectomy.

\section{Materials And Methods}

An acoustic radiation force is a unidirectional force generated by the transfer of the linear momentum from the US compressional wave to the attenuating propagating medium [26]. Because the displacement is directly linked to the amplitude of the radiation force, increasing the displacement magnitude requires optimizing the radiation force efficacy.

As previously mentioned, there is an optimal frequency for the generation of a radiation force. A theoretical model was developed to estimate that frequency depending on the attenuation of the medium and focus depth. For this purpose, the frequency response of the transducer was determined and used to formulate a cost function to determine the underlying parameters of the theoretical model. To support the derivation of that model, the attenuation coefficient was measured for each tested medium using two different methods (a gold standard transmission approach and a spectral shift strategy). This section is presenting the framework of the theoretical model followed by the experimental protocol.

\section{A. Theoretical Modeling}

The amplitude of a focused acoustic radiation force is given by [2], [27]

$$
\begin{gathered}
F=\frac{2 \alpha I \Delta t}{c}, \\
\text { where } I=\frac{P^{2}}{2 \rho c}, \\
\text { and } P=P_{0} e^{-\alpha} .
\end{gathered}
$$

In these equations, $\alpha$ is the wave attenuation, $I$ is the average acoustic intensity, $\Delta t$ is the push duration, $c$ is the compressional wave velocity, $P$ and $P_{0}$ are the attenuated and initial transmitted pressures, respectively, and $\rho$ is the density of the medium. Substituting (2) and (3) into (1) gives the general expression of the radiation force amplitude for a weak diffraction beam:

$$
F=\frac{P_{0}^{2} \Delta t}{\rho c^{2}} \alpha e^{-2 \alpha}
$$

$$
\text { with } \alpha=\alpha_{1} \times f \times d \text {. }
$$

In (5), $\alpha_{1}$ is the attenuation coefficient of the medium, $f$ is the frequency, and $d$ is the focus depth. This latter equation exhibits a local maximum; i.e., that for a certain attenuation coefficient $\left(\alpha_{1}\right)$ and depth $(d)$, there is an optimal frequency $f_{0}$ where the amplitude of the radiation force is maximal. Values of parameters in (4) were varied to estimate the optimal frequency for the generation of the radiation force. They were also determined experimentally, as described below.

\section{B. Experimental Protocol}

1) Determination of the Transducer Frequency Response and Radiation Pressure Intensities: The theoretical model of (4) assumes that the transducer response is constant within the frequency range of interest, which is rarely true. To improve the accuracy of predictions, this model was weighted by the experimentally determined transducer frequency response. Measuring transducer characteristics also allowed the determination of most often used acoustic parameters useful to quantify the deposited energy, such as the maximum intensity ( $\left.\mathrm{I}_{\mathrm{SPPA}}\right)$, average intensity ( $\left.\mathrm{I}_{\mathrm{SPTA}}\right)$, and mechanical index (MI) [28], [29].

As shown in Fig. 1(a), to measure the acoustic pressure generated by the US transducer in a degassed water tank, a hydrophone (HP Series, Precision Acoustics, Dorchester, UK) was positioned at the focus of a linear array transducer (ATL L7-4, Philips, Bothell, WA, USA) connected to a Verasonics scanner (version V1, Verasonics Inc., Redmond, WA, USA). The radiation force was generated at different single excitation frequencies between 2.9 and 6 $\mathrm{MHz}$, which are within frequency values programmable by the Verasonics system for the transducer chosen in this study. The excitation amplitude (transducer voltage) used to generate the radiation force was fixed at $30 \mathrm{~V}$ (peak to peak) throughout all experiments. The burst duration of each excitation sequence was around $100 \mu$ s (the number of cycles varied depending on the frequency, it ranged from 290 to 600 cycles). Acoustic signals were received by the needle hydrophone and synchronously recorded using a digitizer (model Gagescope CS8500, Gage Applied Technologies Inc., Lachine, QC, Canada) after amplification (model 5900PR, Parametrics, Waltham, MA, USA). These signals were converted to pressure values using the hydrophone transfer function provided by the manufacturer. Computed pressures were used to determine acoustic intensities and the MI, as in [29]:

$$
\begin{gathered}
I_{\mathrm{SPPA}}=\frac{P_{\max }^{2}}{2 \rho c}, \\
I_{\mathrm{SPTA}}=I_{\mathrm{SPPA}} \times \frac{\Delta t}{1 s}, \\
\mathrm{MI}=\frac{P r_{\max }}{\sqrt{f_{\mathrm{c}}}},
\end{gathered}
$$




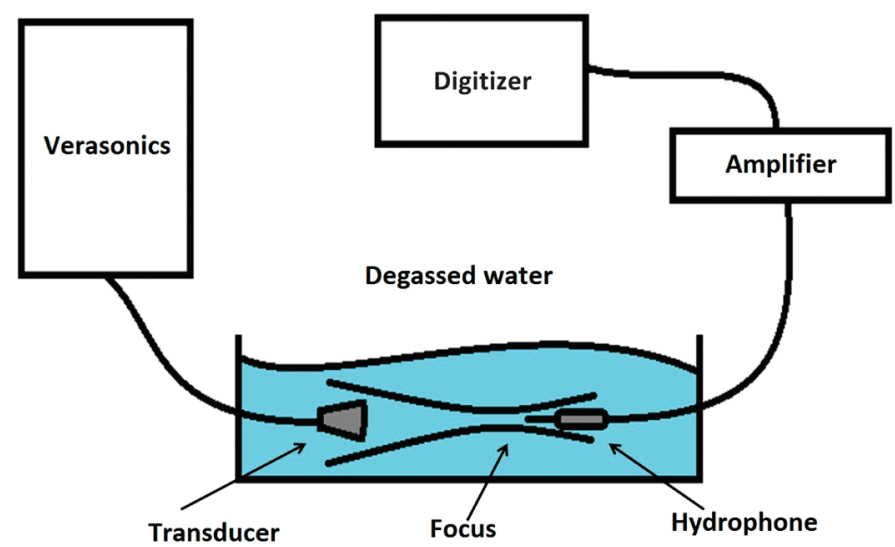

(a)

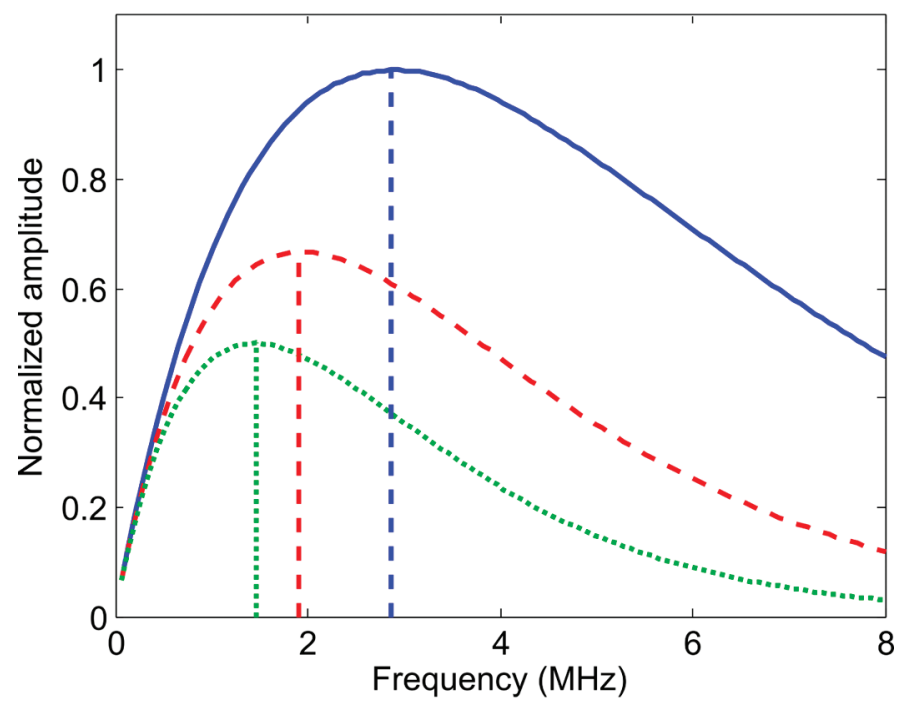

(b)

Fig. 1. (a) Schematic illustration of the experimental setup used for the characterization of the transducer. (b) Theoretical modeling of the amplitude of the radiation force versus frequency in a medium with an attenuation coefficient $\alpha_{1}=0.5 \mathrm{~dB} / \mathrm{cm} / \mathrm{MHz}$ at different focusing depths of $25 \mathrm{~mm}$ (solid blue), $40 \mathrm{~mm}$ (dashed red), and $55 \mathrm{~mm}$ (dotted green). The theoretical model was derived from (4), and the radiation force amplitude was normalized to the greatest maximum.

where $P_{\max }$ and $P r_{\max }$ are the maximum and maximum rarefaction (negative) pressures, respectively, and $f_{\mathrm{c}}$ is the central frequency of the transducer. It is worth mentioning that the excitation voltage at $30 \mathrm{~V}$ avoided performing measurements in the saturation region, which was experimentally determined at $38 \mathrm{~V}$.

2) Fabrication of Phantoms: Four phantoms $(10 \times 10 \times$ $10 \mathrm{~cm}^{3}$ ) were fabricated following the protocol described in [30]. Three of these phantoms were homogenous with different concentrations of agar (product $\mathrm{N}^{\circ} \mathrm{A}-9799$, Sigma-Aldrich, St. Louis, MO, USA) and gelatin (product $\mathrm{N}^{\circ}$ G-2500, Sigma-Aldrich) to get different attenuations, and the other was heterogeneous and made of three different layers. Each layer represented one-third of the total volume of the phantom with a thickness of around $33 \mathrm{~mm}$. Samples were taken from each gel mixture to estimate
TABLE I. Concentrations of Agar and Gelatin Used for Fabricating Each Phantom.

\begin{tabular}{llcc}
\hline & & \multicolumn{2}{c}{ Concentration $(\%)$} \\
\cline { 3 - 4 } Phantom & & Agar & Gelatin \\
\hline Phantom \#1 & 4 & 3 \\
Phantom \#2 & 5 & 4 \\
Phantom \#3 & & 6 & 5 \\
Phantom \#4 & First layer & 6 & 5 \\
& Second layer & 5 & 4 \\
& Third layer & 4 & 3 \\
\hline
\end{tabular}

The proportions are relative to the water weight.

their attenuation coefficients by two different methods described in the next section. Table I summarizes details on concentrations used for the preparation of each phantom. Percentages are in proportion of the water weight.

3) Measurement of Attenuation Coefficients: Harmonic signals (20 cycles, $1 \mathrm{~V}$ peak to peak, frequencies of 3.5, 4, 5 , and $7.5 \mathrm{MHz}$ ) were produced by a function generator (model 33250A, Agilent, Palo Alto, CA, USA) and amplified (model AR 75A250, Amplifier Research, Souderton, PA, USA) before supplying a single element transducer (model IL0506HP, Valpey Fisher Inc., Hopkinton, MA, USA). The acoustic signal emitted by the transducer was measured using the same hydrophone as before (HP Series, Precision Acoustic) without (reference signal) and with phantom samples of 30,45 , and $75 \mathrm{~mm}$ thicknesses in the path. The signal obtained by the hydrophone was amplified (model 5900PR, Parametrics) and digitized (model CS8500, Gage Applied Technologies Inc.). Temporal signals were Fourier transformed to extract the amplitude at the exact frequency of interest.

For each frequency, the attenuation of each sample, $\alpha_{\mathrm{f}}$ $(\mathrm{dB} / \mathrm{cm})$, was calculated using the following equation [31]:

$$
\alpha_{\mathrm{f}}=20 \log _{10}\left(\frac{V_{0}}{V}\right)
$$

where $V_{0}$ is the reference signal (only water in the path) and $V$ is the signal after passing through the sample. A mean attenuation at each frequency (between 3.5 and 7.5 $\mathrm{MHz}), \bar{\alpha}_{\mathrm{f}}$, was then computed from attenuation values of the three thickness samples of each phantom, obtained by (9). Then, the attenuation coefficient was estimated for all pair frequencies, $f_{i}$ and $f_{j}$, using [31]:

$$
\alpha_{i j}=\frac{\bar{\alpha}_{f_{i}}-\bar{\alpha}_{f_{j}}}{f_{i}-f_{j}}
$$

where $\bar{\alpha}_{f_{i}}$ and $\bar{\alpha}_{f_{j}}$ are mean attenuation coefficients at frequencies $f_{i}$ and $f_{j}$, respectively. The attenuation coefficient of the medium, i.e., $\alpha_{1}$ of (5) expressed in $\mathrm{dB} / \mathrm{cm} / \mathrm{MHz}$, was obtained by averaging estimated attenuation coefficients of pair frequencies calculated from (10).

As the latter method cannot be used for heterogeneous media and biological tissue samples, attenuation coeffi- 
cients of all phantoms were also measured by a second method based on a spectral shift approach applied on RF data. This method was also applied on the ex vivo breast tissue sample. It consisted of dividing RF signals of each US beam into short overlapping segments (depending on the depth). The power spectrum was calculated for each segment and fit by a Gaussian function to find the central frequency $f_{\mathrm{c}}$ and the bandwidth $\sigma_{\omega}^{2}$. Then, the attenuation slope $\alpha_{0}$ was estimated from the down shift of the central frequency with depth as [32], [33]:

$$
\alpha_{0}=-\frac{1}{4 \sigma_{\omega}^{2}} \frac{\partial f_{\mathrm{c}}}{\partial z} .
$$

The attenuation coefficient of (5) in $\mathrm{dB} / \mathrm{cm} / \mathrm{MHz}$ was obtained by [34]:

$$
\alpha_{1}=\alpha_{0} f_{c}
$$

Using this method, the measurement of the pressure (intensity) at the focus is not needed for the estimation of the optimal frequency. Indeed, for given transducer voltage affecting the initial pressure $P_{0}$, given excitation duration $(\Delta t)$, and known properties of the medium ( $\rho$ and $c)$, the first term of (4) is a constant and the optimal frequency depends only on the attenuation.

\section{4) Adapted ARFI and SSI Radiation Pressure Strate- gies:}

a) In vitro phantom measurements: Four different sequences were tested (two nonadapted and two adapted) for each phantom. The nonadapted sequences were a home implementation of single push ARFI and SSI [3], [4]. For the remaining of this document, the adapted ARFI sequence is labeled adapted ultrasound push beam (AUPB), whereas the adapted SSI strategy is named adapted ultrasound supersonic beam (AUSB). In contrast to ARFI, in AUPB the frequency for the generation of the radiation force was adapted by considering the attenuation coefficient of the medium and the focus depth. Contrary to the classic SSI scheme where the same frequency is used to generate all focused pushes, in AUSB each push was generated at its optimal frequency (it thus changed as a function of depth).

For each agar-gelatin phantom, the radiation force was generated locally at the central frequency $f_{\mathrm{c}}$ of $5.0 \mathrm{MHz}$ (home ARFI) and at an optimal frequency (AUPB) predicted by the analytical model (4). Radiation forces were produced at focus depths of 25,40 , and $55 \mathrm{~mm}$ in the case of homogeneous phantoms, and 20, 45, and $60 \mathrm{~mm}$ for the heterogeneous phantom \#4. The choice of focus depths between 20 and $45 \mathrm{~mm}$ was based on typical settings for dynamic elastography of breast lesions [35], and the two deeper focus depths (55 and $60 \mathrm{~mm}$ ) were considered to assess the feasibility of the adaptive AUPB method at deeper regions. As used earlier for the determination of the transducer characteristics, the excitation amplitude was fixed at $30 \mathrm{~V}$ and the duration of the focused push was maintained constant at approximately $100 \mu \mathrm{s}$ at all frequencies.

Two supersonic beam sequences (Mach number $=100$ ) were also generated in each phantom. The sequence consisted of 3 focus spots at 25, 40, and $55 \mathrm{~mm}$ for phantoms \#1, \#2, and \#3 (homogeneous), and 20, 45, and $60 \mathrm{~mm}$ for phantom \#4 (heterogeneous). The transducer excitation voltage and duration of focused pushes were the same as used earlier for ARFI and AUPB (30 V and around $100 \mu \mathrm{s}$, respectively). For the first supersonic beam sequence (home SSI), the selected frequency was the central frequency of the transducer that remained the same as a function of depth $(5.0 \mathrm{MHz})$. For the second sequence (AUSB), the pushing beam frequency was adjusted by considering (4).

For all experiments (nonadapted and adapted ARFI and SSI), tracking of shear waves was performed using a fast plane wave imaging method described in [36], allowing retrospective image reconstruction at a frame rate of $3850 \mathrm{~Hz}$. Each sequence was repeated ten times for each phantom, and averaged results are reported.

b) Ex vivo breast sample measurements: To analyze the impact of the adapted frequency strategy with a scope of future clinical applicability, experiments were also done with an ex vivo total mastectomy human breast sample. The sample was provided by the Department of Pathology of the University of Montreal Hospital following approval by the Institutional Human Ethic Committee and patient's informed consent. ARFI and AUPB sequences were not tested; the first sequence was the home SSI implementation and the second one was the AUSB (adapted sequence). The transducer voltage (excitation amplitude) was fixed at $35 \mathrm{~V}$ and the push duration was as for in vitro measurements, at approximately $100 \mu \mathrm{s}$ for all frequencies. The reason for the increase from 30 to $35 \mathrm{~V}$ between phantom and ex vivo experiments was because at $30 \mathrm{~V}$, the shear wave amplitude appeared smaller than phantom data, which could impact the quality of results. We did not use higher voltages to mimic future in vivo tests for which tissue heating may be a concern. The attenuation coefficient needed for the calculation of optimal frequencies for the generation of radiation forces was estimated using the spectral shift method (i.e., adaptive method applicable in vivo). After ultrasound data acquisitions, the breast sample was returned to perform pathology analyses, according to standard clinical practices. As for in vitro measurements, sequences were repeated ten times and averaged results are reported.

c) Shear wave velocity and SNR estimation: To allow comparison of data with the literature, the shear wave velocity was estimated in all phantoms and in the ex vivo breast sample using the time of flight algorithm [4], [5]. The SNR of the shear wave velocity field was also evaluated in two different regions of interest (ROI). For single push ARFI and AUPB, ROIs were at the depth chosen to generate the radiation pressure (between 20 and $60 \mathrm{~mm}$ ), 
at a lateral distance of $2 \mathrm{~mm}$ (from the push location) for the first ROI (ROI \#1) and $20 \mathrm{~mm}$ laterally (from the same push location) for the second ROI (ROI \#2). In the case of SSI and AUSB, ROIs were at the same focus depths of 20 to $60 \mathrm{~mm}$, and at the same lateral position as for ARFI and AUPB (2 and $20 \mathrm{~mm}$ from the push location). Each ROI was defined as a rectangle of $15 \mathrm{~mm}$ (axially) by $10 \mathrm{~mm}$ (laterally). The SNR was computed as the ratio between the mean $(\mu)$ and standard deviation $(\sigma)$ of the shear wave velocity within the ROI using the following equation [22]:

$$
\mathrm{SNR}=\frac{\mu}{\sigma}
$$

\section{RESULTS}

\section{A. Theoretical Modeling}

Fig. 1(b) shows the amplitude of the radiation force in a medium with an attenuation coefficient $\alpha_{1}=0.5 \mathrm{~dB} /$ $\mathrm{cm} / \mathrm{MHz}$ (typical attenuation coefficient of soft biological tissues [37]) for different focusing depths of 25,40 , and 55 $\mathrm{mm}$. It is seen that at each depth there is an optimal frequency where the amplitude of the radiation force would be maximal. It is worth mentioning that at this step, the theoretical model does not take into account the frequency response of the transducer.

\section{B. Experimental Results}

1) Frequency Response of the Transducer: The frequency response of the transducer used in this study is shown in Fig. 2. The central frequency, $f_{c}$, is around $5.0 \mathrm{MHz}$, and the bandwidth at full width half maximum (FWHM) is approximately $3.5 \mathrm{MHz}$. The amplitude was normalized relative to the maximum amplitude at the central frequency. The maximum intensity $\left(\mathrm{I}_{\mathrm{SPPA}}\right)$ for selected firing conditions was $96 \pm 1 \mathrm{~W} \cdot \mathrm{cm}^{-2}$, the average intensity $\left(\mathrm{I}_{\text {SPTA }}\right)$ was $9.6 \pm 0.2 \mathrm{~mW} \cdot \mathrm{cm}^{-2}$, and the MI was $0.27 \pm$ 0.01 . These values are within the acceptable range suggested by the FDA for in vivo applications [38]. The frequency response of the transducer was used to modify the theoretical model for AUPB and AUSB implementations. Fig. 3 shows the radiation force amplitude of Fig. 1(b) weighted by the frequency response of Fig. 2 (considering the same typical attenuation coefficient $\alpha_{1}=0.5 \mathrm{~dB} / \mathrm{cm} /$ $\mathrm{MHz}$ and the same focus depths of 25, 40, and $55 \mathrm{~mm}$ ).

2) Attenuation Coefficients: Table II presents mean and standard deviations of attenuation coefficients obtained for each phantom and the ex vivo breast sample using both methods. Because comparable results were obtained with both approaches and as the spectral shift method is not constrained to homogenous tissue samples, the latter technique was privileged to determine $\alpha$ (4) required to estimate adapted frequencies for AUPB and AUSB elastography methods.

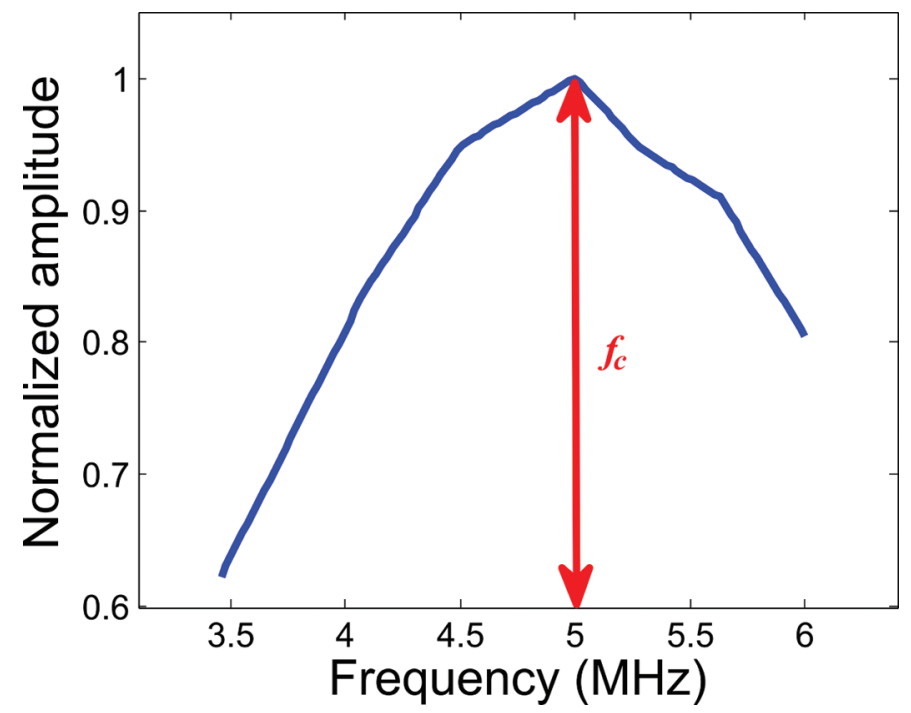

Fig. 2. Experimental transducer frequency response of an ATL L7-4 US probe measured with a needle hydrophone in a degassed water tank. The amplitude was normalized to the maximum response. The parameter $f_{\mathrm{c}}$ represents the central frequency of the transducer.

3) Comparison Between Theoretical Modeling and Experimental Results: Fig. 4(a)-4(d) shows theoretical and experimental (adapted single push ARFI, i.e., AUPB) radiation force normalized amplitudes for each phantom at different focusing depths. The experimental radiation force amplitude was determined using measured displacements, which were estimated very close to the push location (same depth at $0.308 \mathrm{~mm}$ laterally from the push) [24], [25]. Depending on the focus depth and the attenua-

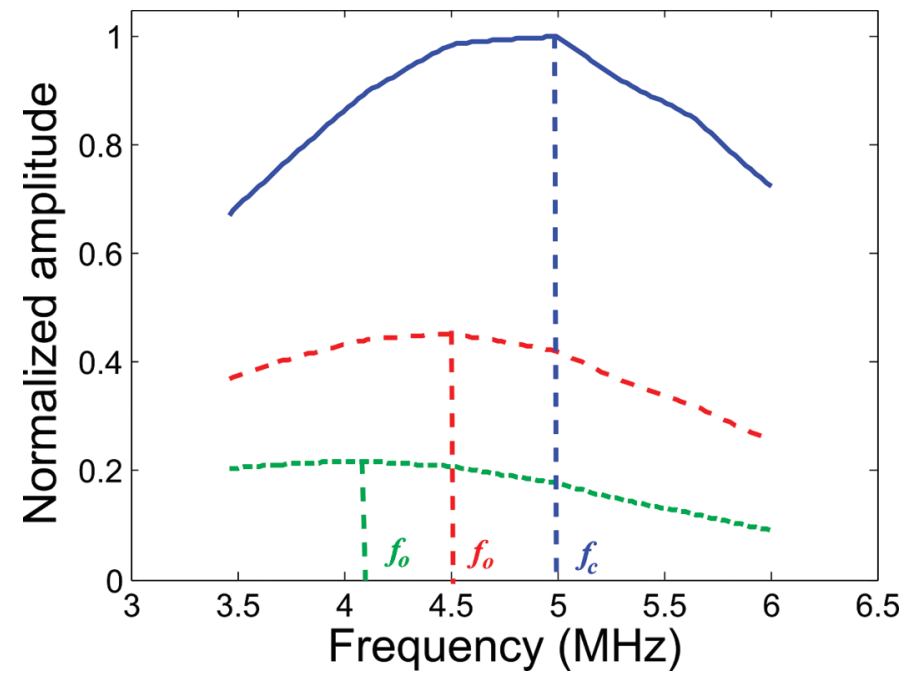

Fig. 3. Normalized amplitudes of the radiation force as a function of frequency for $\alpha_{1}=0.5 \mathrm{~dB} / \mathrm{cm} / \mathrm{MHz}$ at different focusing depths of 25 $\mathrm{mm}$ (solid blue), $40 \mathrm{~mm}$ (dashed red), and $55 \mathrm{~mm}$ (dotted green). The attenuation coefficient and focus depths correspond to values used in Fig. 1. The theoretical model was derived from (4) and weighted by the transducer frequency response of Fig. 2. The amplitude of the radiation force was normalized to the greatest maximum. Parameters $f_{0}$ correspond to the optimum adapted frequencies, whereas $f_{\mathrm{c}}$ is the central frequency of the transducer. 
TABle II. Attenuation Coefficients $\left[\alpha_{1}\right.$ of (5)] of Phantoms and of the Ex Vivo Breast Sample Estimated By Two Different Methods.

\begin{tabular}{|c|c|c|c|c|c|c|c|}
\hline & \multirow[b]{2}{*}{ Phantom \#1 } & \multirow[b]{2}{*}{ Phantom \#2 } & \multirow[b]{2}{*}{ Phantom \#3 } & \multicolumn{3}{|c|}{ Phantom \#4 } & \multirow{2}{*}{$\begin{array}{c}\text { Ex vivo breast } \\
\text { sample }\end{array}$} \\
\hline & & & & Layer 1 & Layer 2 & Layer 3 & \\
\hline $\begin{array}{l}\text { Coefficient }(\# 1) \\
\alpha_{1}(\mathrm{~dB} / \mathrm{cm} / \mathrm{MHz})\end{array}$ & $0.44 \pm 0.018$ & $0.55 \pm 0.031$ & $0.70 \pm 0.034$ & Not applied & Not applied & Not applied & Not applied \\
\hline $\begin{array}{l}\text { Coefficient }(\# 2) \\
\alpha_{1}(\mathrm{~dB} / \mathrm{cm} / \mathrm{MHz})\end{array}$ & $0.43 \pm 0.027$ & $0.57 \pm 0.038$ & $0.74 \pm 0.041$ & $0.44 \pm 0.029$ & $0.66 \pm 0.044$ & $0.78 \pm 0.049$ & $0.66 \pm 0.051$ \\
\hline
\end{tabular}

Coefficient \#1: hydrophone method; coefficient \#2: spectral shift method.

tion coefficient of the phantom, one can see that the optimal frequency $\left(f_{\mathrm{o}}\right)$ can be similar (e.g., phantoms \#1 and \#2 at $25 \mathrm{~mm}$, and phantom \#4 at $20 \mathrm{~mm}$ ) or different from the central frequency $\left(f_{\mathrm{c}}\right)$ of the transducer. In the case of phantom \#1 (40 and $55 \mathrm{~mm})$, phantom \#2 (40 $\mathrm{mm})$, and phantom \#3 $(25 \mathrm{~mm})$, the optimal frequency was close to $4.5 \mathrm{MHz}$. For phantom \#2 $(55 \mathrm{~mm})$, phantom \#3 (40 mm), and phantom \#4 (45 mm), $f_{\mathrm{o}}$ was $4.09 \mathrm{MHz}$. Finally, for phantom \#3 $(55 \mathrm{~mm})$ and phantom \#4 (60 $\mathrm{mm}$ ), the optimal frequency was lower at $3.46 \mathrm{MHz}$. For

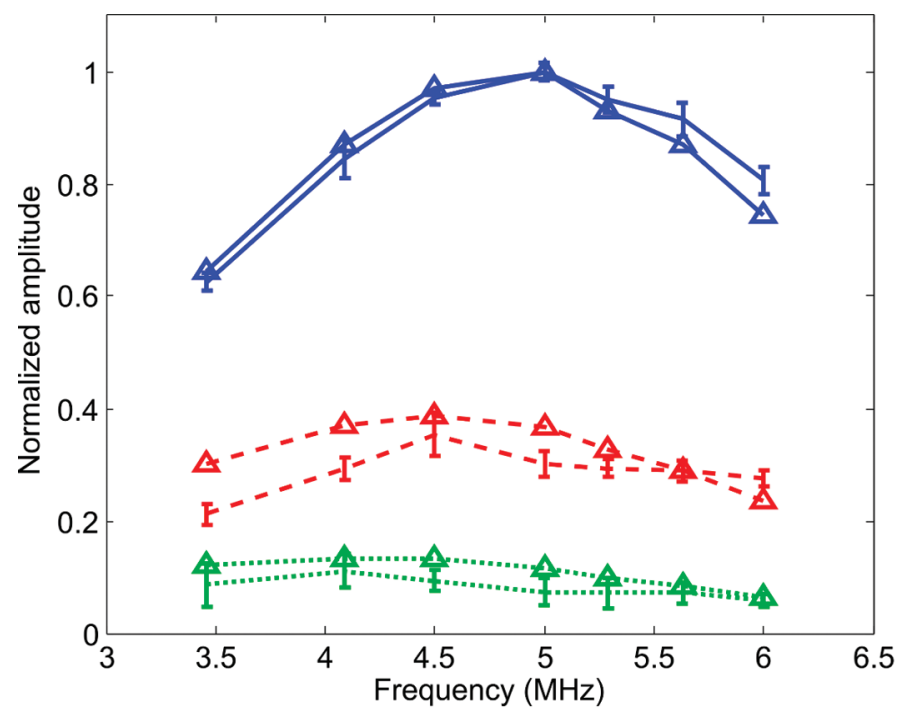

(a)

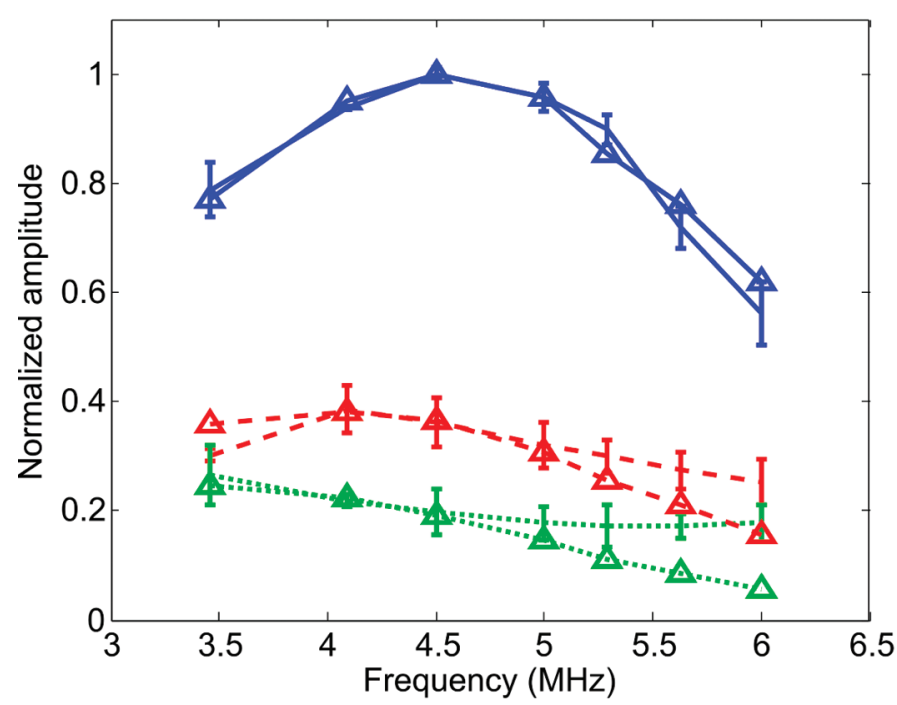

(c)

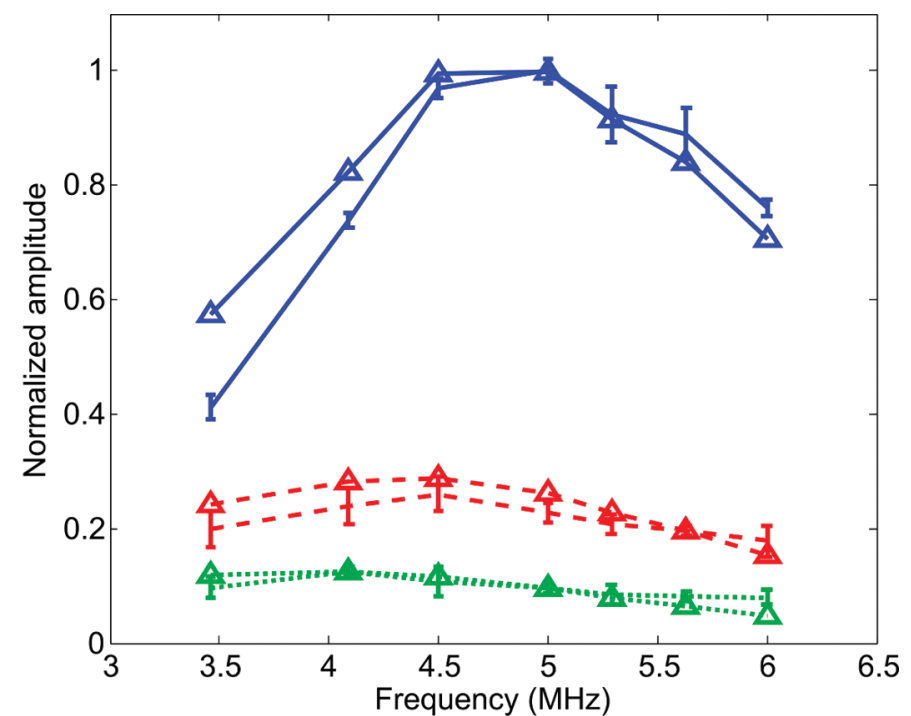

(b)



(d)

Fig. 4. Simulated $(\Delta)$ and experimental (I) radiation force amplitudes versus frequency in phantom \#1 (a), phantom \#2 (b), phantom \#3 (c), and phantom \#4 (d) at different focus depths of $25 \mathrm{~mm}$ (solid blue), $40 \mathrm{~mm}$ (dashed red), and $55 \mathrm{~mm}$ (dotted green). The focus depths correspond to values used in Figs. 1 and 3. The theoretical model was derived from (4) and weighted by the transducer frequency response. The amplitude of the radiation force was normalized to the greatest maximum. Data on this figure correspond to the adapted ARFI method (i.e., AUPB). 


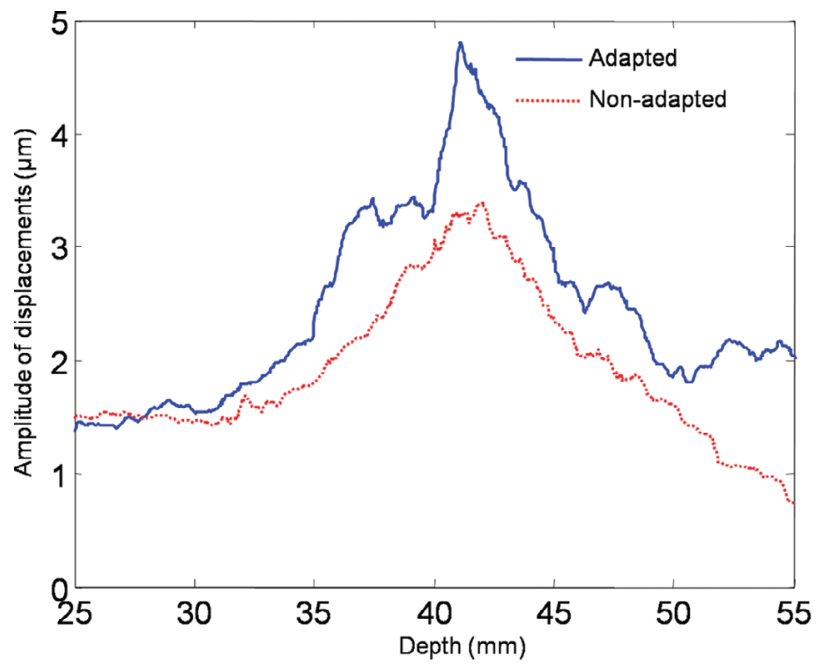

(a)

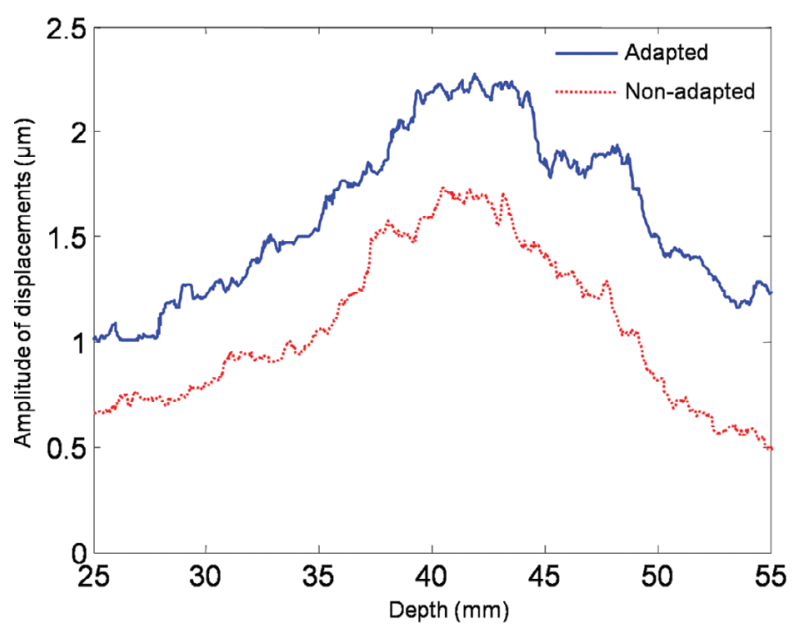

(c)

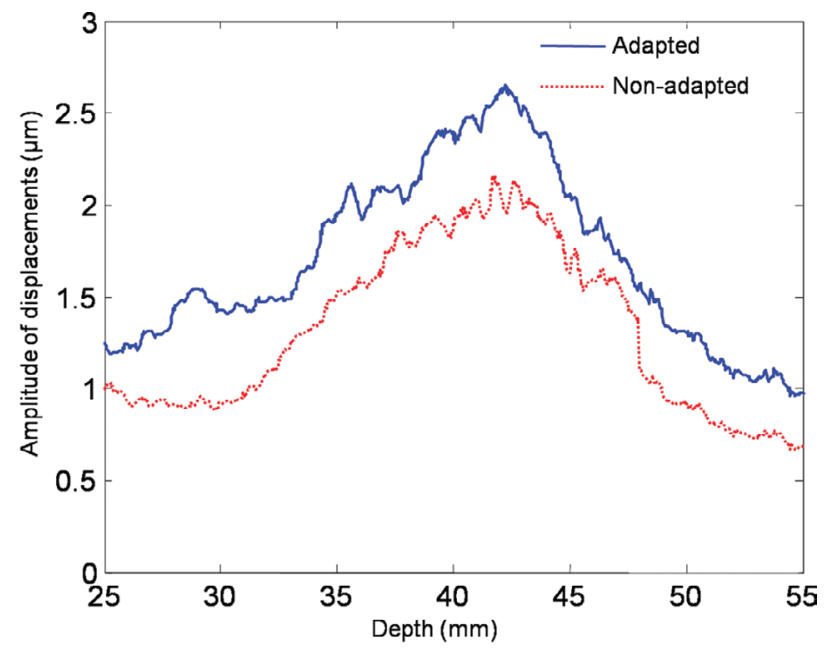

(b)



(d)

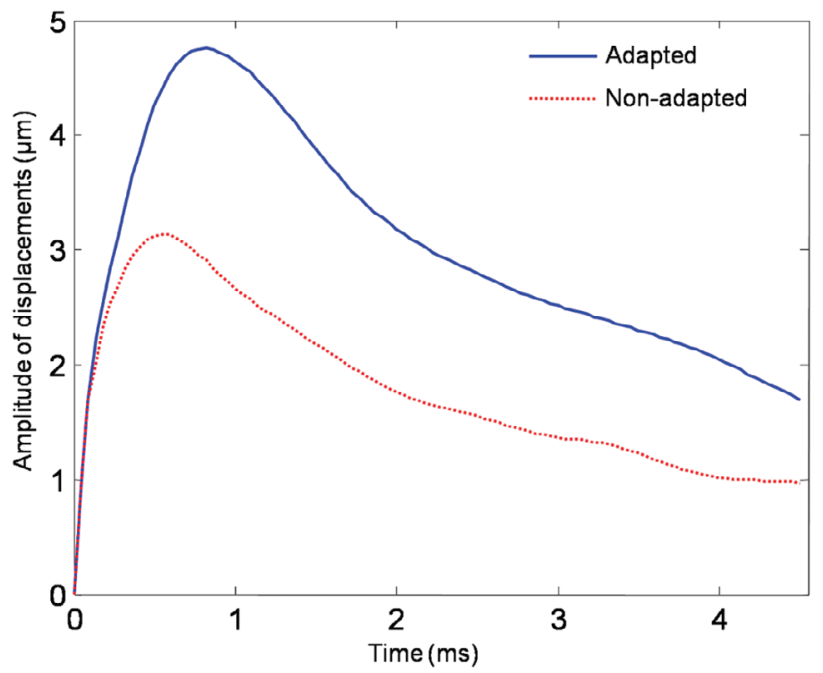

(e)

Fig. 5. Comparison of the amplitude of displacements generated by the adapted ultrasound push beam (AUPB; solid blue) at $40 \mathrm{~mm}$ ( $45 \mathrm{~mm}$ for phantom \#4), and the nonadapted sequence (home ARFI; dashed red) in phantom \#1 (a), phantom \#2 (b), phantom \#3 (c), and phantom \#4 (d). Displacements were estimated at different depths between 25 and $55 \mathrm{~mm}$ (36 to $52 \mathrm{~mm}$ for phantom \#4), at a lateral distance of $10 \mathrm{~mm}$ from the push location. Measurements were repeated ten times and mean values are shown (standard deviations are not displayed for visibility). (e) Temporal behavior of the displacement generated at the focus push $(40 \mathrm{~mm}$ ) with adapted (solid blue) and nonadapted (dashed red) sequences in phantom \#1. 
TABLE III. Optimal Frequencies and Corresponding Gains in the Amplitude of the Radiation Force for Homogeneous and Heterogeneous Phantoms, and for the Ex Vivo Breast Sample at Different Focus Depths ( $N=10$ Measures $)$.

\begin{tabular}{lcccc}
\hline $\begin{array}{l}\text { Adapted } \\
\text { sequence }\end{array}$ & Medium & Depth $(\mathrm{mm})$ & $\begin{array}{c}\text { Optimal } \\
\text { frequency } f_{0}(\mathrm{MHz})\end{array}$ & Gain $(\%)$ \\
\hline AUPB & Phantom \#1 & 40 & 4.50 & $25 \pm 2$ \\
& Phantom \#2 & 40 & 4.50 & $26 \pm 1$ \\
& Phantom \#3 & 40 & 4.09 & $36 \pm 3$ \\
& Phantom \#4 & 45 & 4.09 & $45 \pm 3$ \\
AUSB & Phantom \#1 & 25 & 5.00 & $21 \pm 2$ \\
& & 40 & 4.50 & $43 \pm 4$ \\
& Phantom \#2 & 55 & 4.50 & $95 \pm 7$ \\
& 25 & 5.00 & $23 \pm 1$ \\
& & 40 & 4.50 & $60 \pm 4$ \\
& & 55 & 4.09 & $114 \pm 6$ \\
& Phantom \#3 & 25 & 4.50 & $28 \pm 2$ \\
& 40 & 4.09 & $62 \pm 4$ \\
& Phantom \#4 & 55 & 3.46 & $133 \pm 5$ \\
& 20 & 5.00 & $20 \pm 1$ \\
& & 45 & 3.09 & $106 \pm 5$ \\
& & 60 & 3.46 & $158 \pm 6$ \\
& & 20 & 5.00 & $170 \pm 11$ \\
& & 25 & 4.50 & $290 \pm 13$ \\
& 30 & 4.50 & $336 \pm 18$ \\
\hline
\end{tabular}

For AUPB, gains were calculated at the same depth as the focus (40 $\mathrm{mm}$ for homogeneous phantoms and $45 \mathrm{~mm}$ for the heterogeneous one) at a lateral distance of $10 \mathrm{~mm}$ from the push. In case of AUSB, gains were estimated at each focus depth at the same lateral distance as for AUPB $(10 \mathrm{~mm})$.

the remainder of this document, the gain in magnitude for each focus depth is expressed as the ratio of the amplitude of displacements at adapted frequencies $f_{\mathrm{O}}$ to that at the transducer central frequency $f_{\mathrm{c}}$ :

$$
\text { gain }=\frac{\text { amplitude at } f_{\mathrm{o}}}{\text { amplitude at } f_{\mathrm{c}}} \times 100 \text {. }
$$

4) Performance Assessment of $A U P B$ and $A U S B$ Sequences: Figs. 5(a) to 5(d) present the amplitude of displacements generated by AUPB and nonadapted ARFI push beams in homogeneous and heterogeneous phantoms, at a focus depth of $40 \mathrm{~mm}$ ( $45 \mathrm{~mm}$ for the heterogeneous phantom), between 25 and $55 \mathrm{~mm}$ within each phantom (36 to $52 \mathrm{~mm}$ for phantom \#4) at a lateral distance of $10 \mathrm{~mm}$ from the push. As seen, displacement amplitudes along the push beam generated by the AUPB method were greater than those obtained by the nonadapted sequence. Depending on the attenuation coefficient of the phantom, gains (14) at a depth of $40 \mathrm{~mm}$ were $25 \pm 2 \%$ for the softest phantom (phantom \#1), $26 \pm 1 \%$ for the intermediate one (phantom \#2), and $36 \pm 3 \%$ for the stiffest medium (phantom \#3). In the case of the heterogeneous phantom, the gain at a depth of $45 \mathrm{~mm}$ was $45 \pm 3 \%$ (see Table III).
Fig. 5(e) shows a comparison of the temporal behavior of the displacement at the focus $(40 \mathrm{~mm})$ generated at the central frequency of the transducer and at the optimal frequency in phantom \#1.

Figs. 6(a) to 6(d) show the comparison between the amplitude of displacements generated in phantom samples by AUSB and the nonadapted supersonic beam (home SSI) along the plane wave front between 20 to $60 \mathrm{~mm}$ for homogeneous phantoms, and 10 to $63 \mathrm{~mm}$ for the heterogeneous one at a lateral distance of $10 \mathrm{~mm}$ from the push line. Again, the amplitude of displacements generated by the adapted sequence was greater at any depth. Gains varied between $20 \pm 1 \%$ and $158 \pm 6 \%$ depending on the focus depth and the attenuation coefficient of the tested medium. For phantom $\# 1$, gains were $21 \pm 2 \%$ for the first focus, $43 \pm 3 \%$ for the second focus, and $95 \pm 7 \%$ for the third focus. In the case of phantom \#2, gains were $23 \pm 1 \%, 60 \pm 4 \%$, and $114 \pm 6 \%$ for focus depths at 25 , 40, and $55 \mathrm{~mm}$, respectively. For phantoms \#3 and \#4, gains were $28 \pm 2 \%$ and $20 \pm 1 \%$ for the first focus, 62 $\pm 4 \%$ and $106 \pm 5 \%$ for the second focus, and $133 \pm 5 \%$ and $158 \pm 6 \%$ for the third focus, respectively (see Table III). The gain in magnitude was greater than phantom data (between $170 \pm 11 \%$ and $336 \pm 18 \%$ ) when using the

Table IV. Mean Shear Wave Velocities (in Meters Per Second) and Their Corresponding Standard Deviations in Different Phantoms and in the EX VIVo Breast Sample Using Adapted and Nonadapted SSi Sequences.

\begin{tabular}{|c|c|c|c|c|c|c|c|}
\hline \multirow{2}{*}{$\begin{array}{l}\text { Mean velocity } \pm \\
\mathrm{SD}(\mathrm{m} / \mathrm{s})\end{array}$} & \multirow[b]{2}{*}{ Phantom \#1 } & \multirow[b]{2}{*}{ Phantom \#2 } & \multirow[b]{2}{*}{ Phantom \#3 } & \multicolumn{3}{|c|}{ Phantom \#4 } & \multirow{2}{*}{$\begin{array}{l}\text { Ex vivo } \\
\text { sample }\end{array}$} \\
\hline & & & & Layer 1 & Layer 2 & Layer 3 & \\
\hline Adapted sequence & $2.8 \pm 0.3$ & $3.8 \pm 0.3$ & $5.9 \pm 0.4$ & $6.4 \pm 0.3$ & $5.3 \pm 0.4$ & $2.4 \pm 0.6$ & $2.1 \pm 0.1$ \\
\hline Nonadapted sequence & $2.9 \pm 0.4$ & $3.9 \pm 0.4$ & $6.2 \pm 0.7$ & $6.3 \pm 0.5$ & $4.6 \pm 0.8$ & $1.8 \pm 0.9$ & $1.8 \pm 0.6$ \\
\hline
\end{tabular}




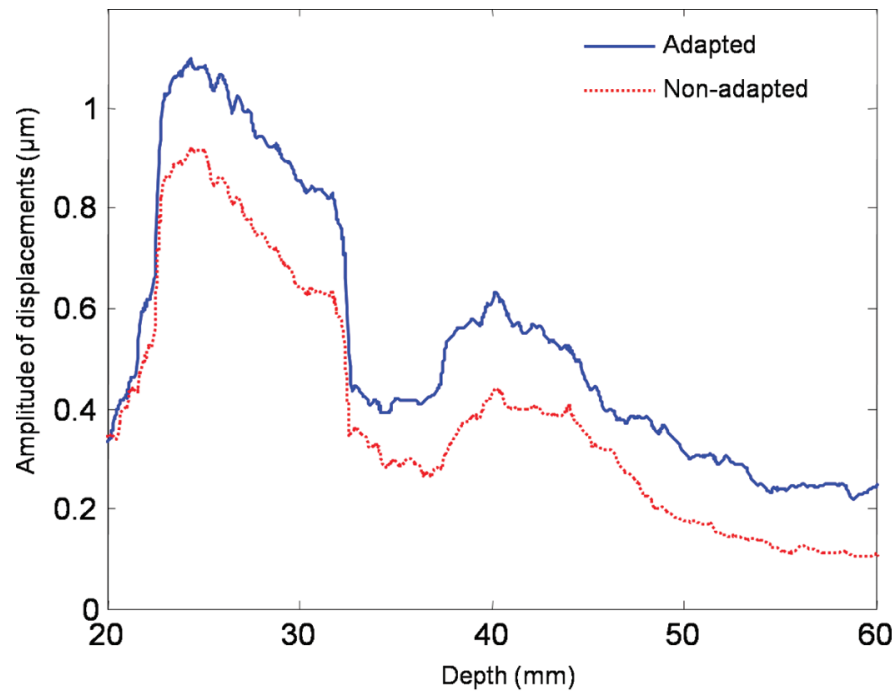

(a)

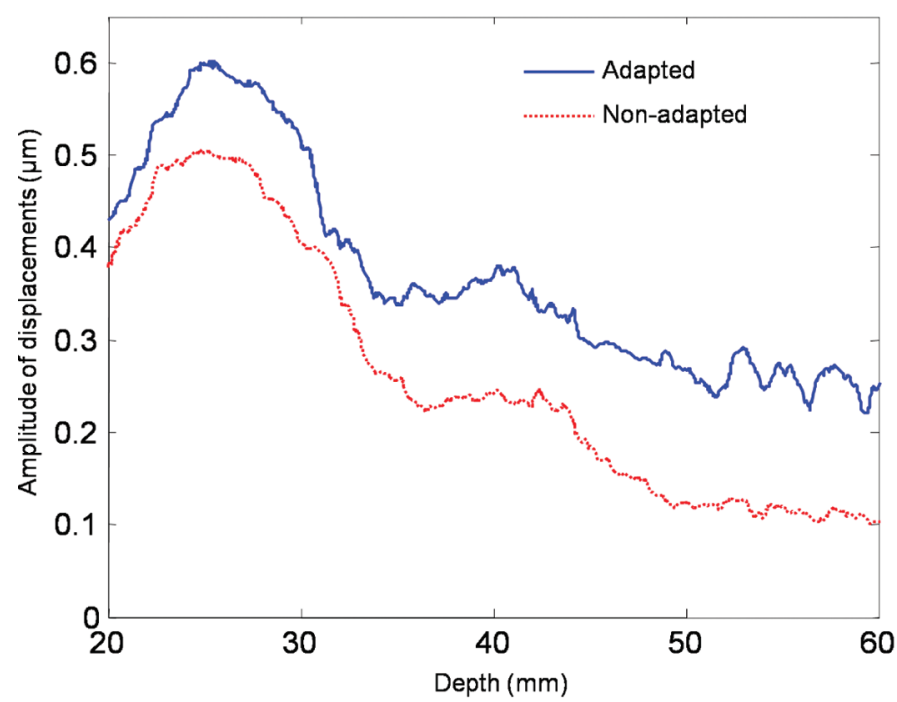

(c)

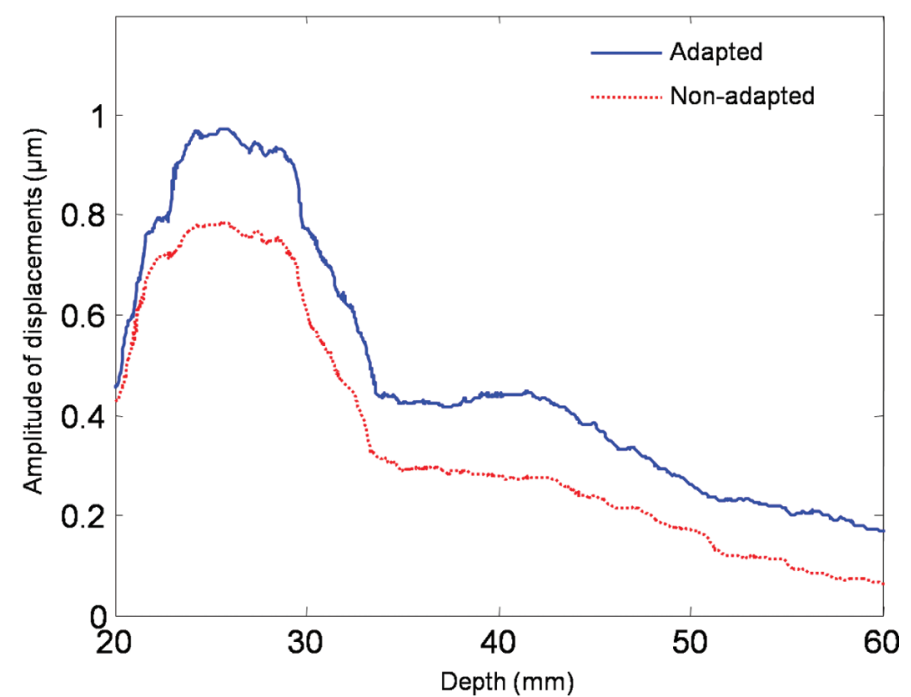

(b)

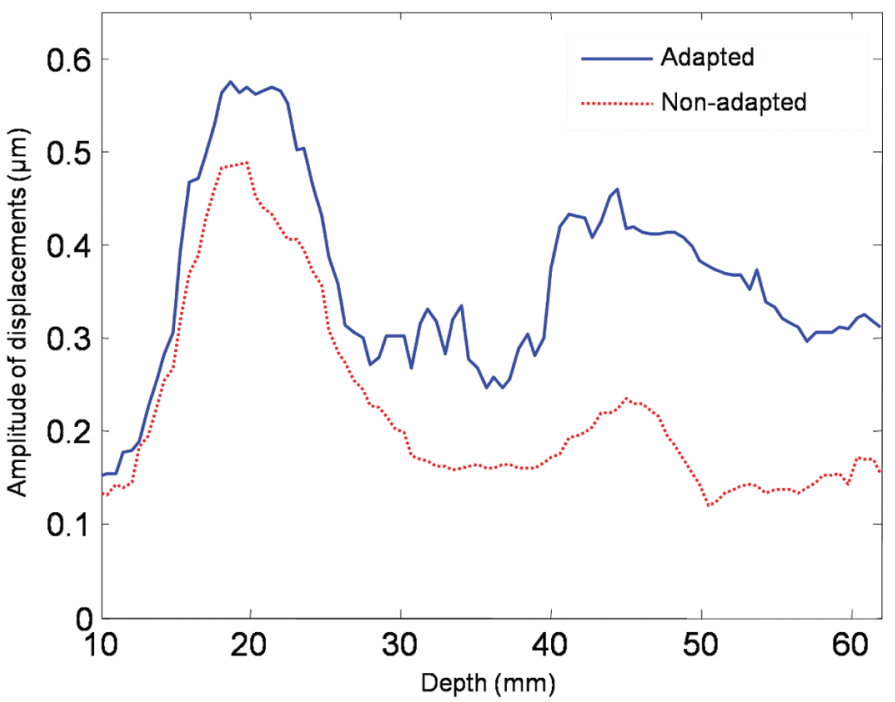

(d)

Fig. 6. Comparison of the amplitude of displacements generated by the adapted ultrasound supersonic beam (AUSB; solid blue) and the nonadapted sequence (home SSI; dashed red) generated at 25, 40, and $55 \mathrm{~mm}$ in phantom \#1 (a), phantom \#2 (b), and phantom \#3 (c), and at $20 \mathrm{~mm}$ (soft layer), $45 \mathrm{~mm}$ (medium layer), and $60 \mathrm{~mm}$ (hard layer) in phantom \#4 (d). Displacements were estimated along the plane wave front at depths of 20 to $60 \mathrm{~mm}$ for homogeneous phantoms, and between 10 to $63 \mathrm{~mm}$ for the heterogeneous one at a lateral distance of $10 \mathrm{~mm}$ from the push line. Measurements were repeated ten times, and mean values are shown (standard deviations are not displayed for visibility).

adapted AUSB sequence in the case of the ex vivo breast sample (see Fig. 7 and Table III).

As presented in Table IV, shear wave velocities estimated with AUSB were close to those of the nonadapted SSI sequence, but overall, standard deviations were smaller in the case of the adapted sequence (i.e., AUSB). For the ex vivo breast sample, the velocity obtained with the adapted sequence was $2.1 \pm 0.1 \mathrm{~m} / \mathrm{s}$, whereas for the nonadapted sequence it was $1.8 \pm 0.6 \mathrm{~m} / \mathrm{s}$.

The SNR of adapted (AUSB) and nonadapted (home SSI) sequences in two different ROIs for each homogeneous phantom are shown in Fig. 8(a). Figs. 8(b) and 8(c) show the SNR with both sequences in different layers of the heterogeneous phantom, and in the case of the ex vivo human breast sample, respectively. It is seen that the SNR varies less in ROI \#1 (close to the focus push) than ROI \#2 (distant from the push) between adapted and nonadapted sequences. In the case of homogeneous phantoms and ROI \#1, the SNR was between 20 and 24 $\mathrm{dB}$ for adaptive sequences, and between 15 and $22 \mathrm{~dB}$ for standard sequences. For the second ROI, the SNR was between 23 and $15 \mathrm{~dB}$ for adaptive sequences, and between 17 and $6 \mathrm{~dB}$ for nonadapted ones. In the case of the heterogeneous phantom, the SNR degraded as a function of depth (i.e., for deeper layers 2 and 3), and was still better for the adapted sequence. For the ex vivo sample, the SNR was improved from 16 to $21 \mathrm{~dB}$ in ROI \#1 by using the adapted AUSB sequence, and from 4 to $14 \mathrm{~dB}$ in ROI \#2. Table V summarizes SNR values for all phantoms and for the ex vivo breast sample. 


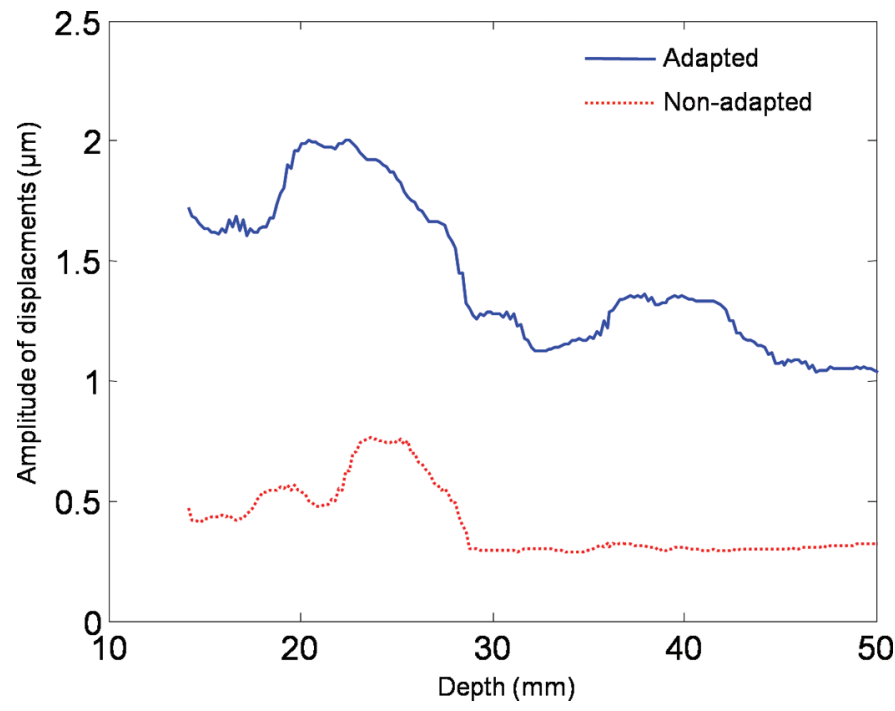

Fig. 7. Comparison of the amplitude of displacements generated by the adapted ultrasound supersonic beam (AUSB; solid blue) and the nonadapted sequence (home SSI; dashed red) generated at 25, 30, and 40 $\mathrm{mm}$ in the ex vivo human breast sample. Displacements were estimated along the plane wavefront at depths between 15 and $50 \mathrm{~mm}$, and at a lateral distance of $10 \mathrm{~mm}$ from the push line. Measurements were repeated ten times, and mean values are shown (standard deviations are not displayed for visibility).

\section{Discussion}

\section{A. Theoretical Modeling}

The radiation force is usually generated at the central frequency of the transducer without taking into account the attenuation of the medium and the focus depth. The theoretical model developed in this study predicted the optimal frequency for the generation of enhanced radiation force by considering these latter two parameters (4). One limitation of this model is that it does not take the frequency response of the transducer into account. To improve the accuracy, the model was weighted by the transducer frequency response (Philips ATL L7-4 in this case). It yielded a theoretical model for the estimation of the optimal frequency for the transducer used in this study. As seen in Fig. 4, theoretical and experimental results were in very good agreement. Therefore, we can conclude that this model yielded good predictions of the optimal frequency for the generation of a radiation force.

\section{B. Experimental Results}

The amplitude of displacements was on average enhanced by 25 to $45 \%$ when comparing the adapted push beam AUPB with the nonadapted ARFI method, at a focus depth of 40 or $45 \mathrm{~mm}$ (Table III). At the same depths, gains in displacement magnitude were greater for AUSB versus SSI (43 to 106\%). This can be explained by the fact that contrary to AUPB for which only one focus push was optimized, the transmitted frequency for AUSB was chosen optimally at each focus depth, thus enhancing the whole wave front by constructive interferences between shear wave sources, thus creating a cumulative effect [4]. According to Table III, interesting observations can be made from comparisons of AUSB versus SSI results in the case of the heterogeneous phantom \#4. Indeed, the displacement gain for the first focus, which was inside the softest layer, was relatively small (20\%). For the second focus inside the medium stiffness layer, the gain was more important (106\%), whereas it was maximum (158\%) for the third focus in the stiffest layer. This can be explained by the greater attenuations in those bottom layers (see Table II), and by the deepest focuses increasing $\alpha$ in (5). As the impact of the frequency adaptation strategy was more important on SSI than on single push ARFI, we opted to apply only AUSB on the ex vivo breast sample, and to compare it to the home implementation of SSI (Table III and Fig. 7).

In this study, we did not compare results to those obtained by the method of Elkateb-Hachemi et al. [25], which consisted in increasing the push duration and transducer voltage, due to the risk of damaging the sample material before histopathology [39]. In [25], authors selected high intensity focused ultrasound (HIFU) transducers that are typically used for thermal therapy. This type of transducers is designed to withstand high voltage amplitudes and long pulse durations [40]. Contrary to [25], we used an imaging transducer (ATL L7-4) for the generation of the radiation force, which is not a power transducer.

When designing this study, we opted to test two different methods for estimating attenuation coefficients $\alpha_{1}$ (5). Because the first method requiring the use of a hydrophone (gold standard) can difficulty be applied to complex heterogeneous media with varying attenuations (i.e., most in vivo measurements), a second well-accepted method

Table V. Estimated SNR (in Decibels) of the Shear Wave Velocity Field and Their Corresponding Standard Deviations in Two Different ROIs (ROI \#1 and ROI \#2) Computed With Adapted (AUSB) and Nonadapted (Home SSI) Sequences for Homogeneous and Heterogeneous Phantoms, and for the EX Vivo Breast Sample.

\begin{tabular}{|c|c|c|c|c|c|c|c|}
\hline \multirow{2}{*}{$\begin{array}{l}\text { Mean SNR } \pm \\
\text { SD }(\mathrm{dB})\end{array}$} & \multirow[b]{2}{*}{ Phantom \#1 } & \multirow[b]{2}{*}{ Phantom \#2 } & \multirow[b]{2}{*}{ Phantom \#3 } & \multicolumn{3}{|c|}{ Phantom \#4 } & \multirow{2}{*}{$\begin{array}{l}\text { Ex vivo } \\
\text { sample }\end{array}$} \\
\hline & & & & Layer 1 & Layer 2 & Layer 3 & \\
\hline Adapted ROI \#1 & $23.3 \pm 1.1$ & $24.2 \pm 1.0$ & $20.2 \pm 1.2$ & $25.6 \pm 1.1$ & $16.4 \pm 1.4$ & $15.1 \pm 1.6$ & $20.9 \pm 1.3$ \\
\hline Nonadapted ROI \#1 & $22.5 \pm 1.2$ & $20.2 \pm 1.3$ & $15.4 \pm 1.5$ & $24.8 \pm 1.2$ & $12.5 \pm 2.1$ & $7.3 \pm 3.3$ & $16.3 \pm 1.7$ \\
\hline Adapted ROI \#2 & $22.3 \pm 1.4$ & $22.9 \pm 1.1$ & $15.8 \pm 1.5$ & $17.0 \pm 1.6$ & $11.8 \pm 2.1$ & $4.3 \pm 3.9$ & $14.2 \pm 2.4$ \\
\hline Nonadapted ROI \#2 & $17.0 \pm 2.0$ & $16.5 \pm 2.7$ & $6.6 \pm 3.2$ & $16.4 \pm 1.6$ & $4.2 \pm 3.7$ & $1.2 \pm 5.5$ & $3.8 \pm 3.4$ \\
\hline
\end{tabular}

ROIs were defined as a rectangle of $15 \mathrm{~mm}$ (axially) by $10 \mathrm{~mm}$ (laterally), and positioned at the same depth of the focus push at lateral distances of 2 and $20 \mathrm{~mm}$ (from the push location) for ROI \#1 and ROI \#2, respectively. 
m Adapted ROI 1 르 Non-adapted ROI 1 Adapted ROI 2 Non-adapted ROI 2

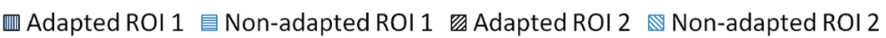

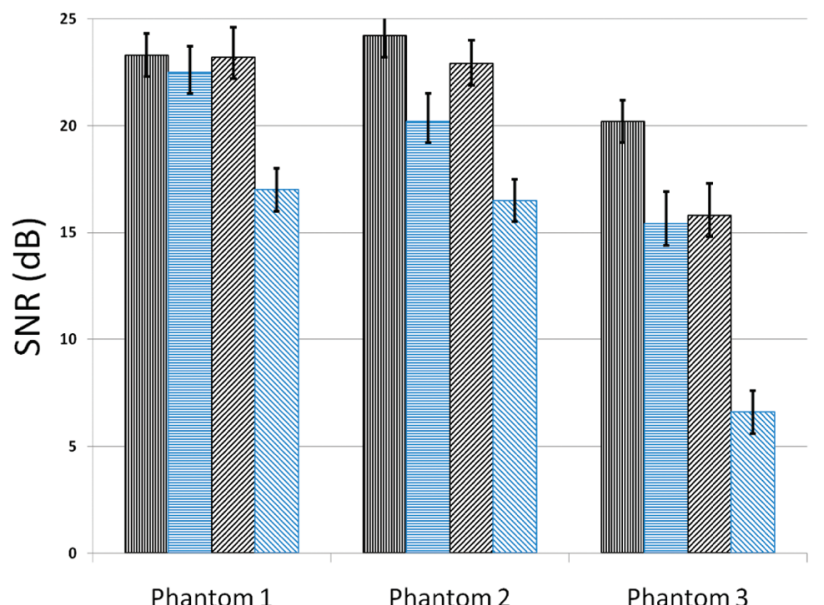

(a)

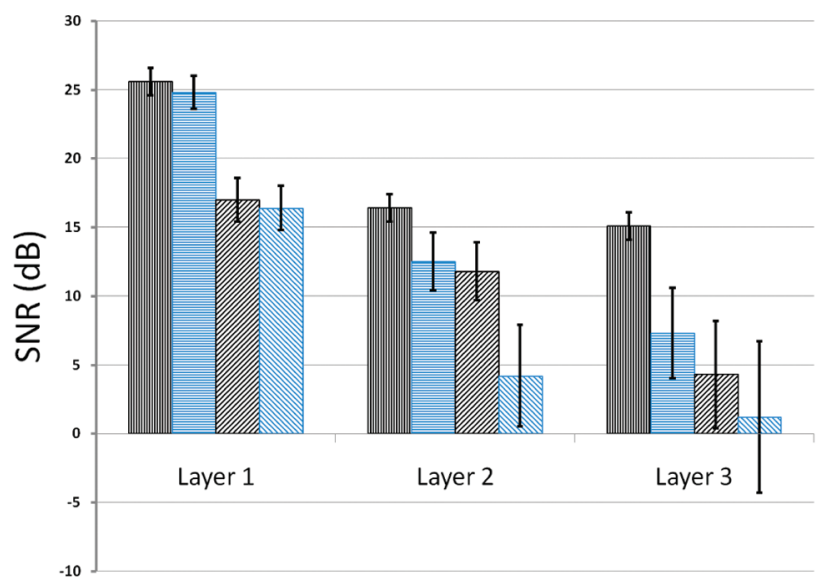

(b)

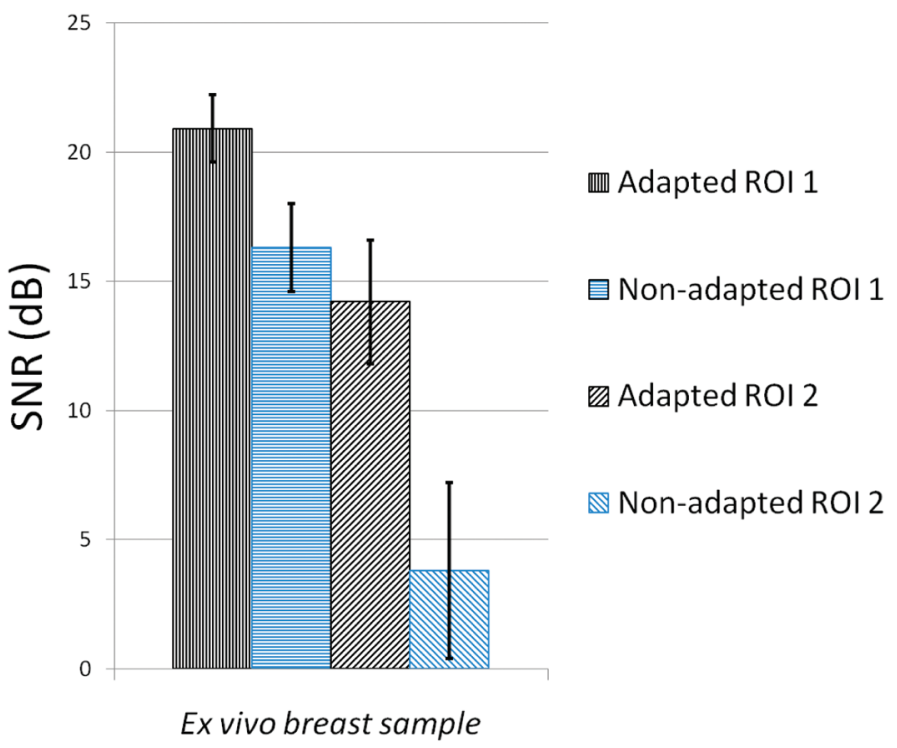

(c)

Fig. 8. SNR of the shear wave velocity field and corresponding standard deviations in two different ROIs (ROI \#1 and ROI\#2) computed for adapted (AUSB) and nonadapted (home SSI) sequences in the case of homogeneous phantoms (a), the heterogeneous phantom (b), and the ex vivo breast sample (c). ROIs were defined as a rectangle of $15 \mathrm{~mm}$ (axially) by $10 \mathrm{~mm}$ (laterally) that was positioned at the same depth of the focus push at lateral distances of 2 and $20 \mathrm{~mm}$ (from the push location) for ROI \#1 and ROI \#2, respectively. Vertical (black) and horizontal (blue) lines represent SNRs of ROI \#1 obtained for adapted and nonadapted sequences, respectively, and oblique lines signify SNRs of ROI \#2 obtained for adapted (right tilted-black) and nonadapted (left tilted-blue) sequences, respectively.

(spectral shift method) based on the down shift of the central frequency was implemented. This method does not require the use of any extra instrumentation and has been clinically validated [41], [42]. Both methods gave similar results (Table II), thus justifying the use of the spectral approach to determine optimal frequencies with (4) for the generation of radiation pressures. For future clinical use, the protocol may be implemented as follows. First, a $\mathrm{B}$ mode image is acquired for the localization of the pushing region. Then, the attenuation at the focus push is estimated directly from the RF data using the spectral shift method, and the optimal frequency computed by the prediction model. Finally, the adapted radiation force is generated and shear waves are tracked using ultrafast plane wave imaging.

Overall, the theoretical model of (4) yielded good predictions of the optimal frequency (see Fig. 4), and shear wave velocities were in good agreement to those obtained for agar-gelatin phantoms with similar concentrations [30]. Velocities for adapted and nonadapted sequences (see Table IV) were similar except for the second and third layers of the heterogeneous phantom, and to a lesser extent for the ex vivo breast sample. This observation may be due to low SNR in these samples [see Figs. 8(b)-8(c) and Table V], which likely affected the quality of the RF signal tracking. It is noticeable that shear wave velocities within 
the ex vivo breast sample were in good agreement with those found in the literature [5], [43].

\section{CONCLUSiON}

Frequency adaptation is a complementary technique that may be used for the optimization of displacement amplitude in ultrasound elastography. This technique can be used safely to optimize the deposited local energy, without the risk of damaging the tissue or the transducer. This technique improves the SNR of the shear wave velocity field for better estimations of mechanical parameters. We found that in all phantoms and in the ex vivo breast sample, regardless of their attenuation or the focus depth, the SNR obtained with adapted sequences were better than those measured using standard sequences. The gain in displacement magnitudes with adapted sequences varied between 20 and 336\%; the benefit of using the SSI adapted sequence was particularity evident in the case of the heterogeneous breast tissue sample.

\section{REFERENCES}

11 A. P. Sarvazyan, O. V. Rudenko, and W. L. Nyborg, "Biomedical applications of radiation force of ultrasound: Historical roots and physical basis," Ultrasound Med. Biol., vol. 36, no. 9, pp. 1379-1394, 2010.

[2] A. P. Sarvazyan, O. V. Rudenko, S. D. Swanson, J. B. Fowlkes, and S. Y. Emelianov, "Shear wave elasticity imaging: A new ultrasonic technology of medical diagnostics," Ultrasound Med. Biol., vol. 24, no. 9, pp. 1419-1435, 1998.

[3] K. R. Nightingale, M. Palmeri, R. W. Nightingale, and G. E. Trahey, "On the feasibility of remote palpation using acoustic radiation force," J. Acoust. Soc. Am., vol. 110, no. 1, pp. 625-634, 2001.

[4] J. Bercoff, M. Tanter, and M. Fink, "Supersonic shear imaging: A new technique for soft tissue elasticity mapping," IEEE Trans. Ultrason. Ferroelectr. Freq. Control, vol. 51, no. 4, pp. 396-409, 2004.

[5] M. Tanter, J. Bercoff, A. Athanasiou, T. Deffieux, J.-L. Gennisson, G. Montaldo, M. Muller, A. Tardivon, and M. Fink, "Quantitative assessment of breast lesion viscoelasticity: Initial clinical results using supersonic shear imaging," Ultrasound Med. Biol., vol. 34, no. 9, pp. 1373-1386, 2008.

[6] V. Landoni, V. Francione, S. Marzi, K. Pasciuti, F. Ferrante, E. Saracca, M. Pedrini, L. Strigari, M. Crecco, and A. Di Nallo, "Quantitative analysis of elastography images in the detection of breast cancer," Eur. J. Radiol., vol. 81, no. 7, pp. 1527-1531, 2012.

7] W. A. Berg, D. O. Cosgrove, C. J. Doré, F. K. W. Schäfer, W. E. Svensson, R. J. Hooley, R. Ohlinger, E. B. Mendelson, C. BaluMaestro, M. Locatelli, C. Tourasse, B. C. Cavanaugh, V. Juhan, A. T. Stavros, A. Tardivon, J. Gay, J.-P. Henry, and C. Cohen-Bacrie, "Shear wave elastography improves the specificity of breast US: The BE1 multinational study of 939 masses," Radiology, vol. 262, no. 2, pp. 435-449, 2012.

[8] X. Chen, Y. Shen, Y. Zheng, H. Lin, Y. Guo, Y. Zhu, X. Zhang, T. Wang, and S. Chen, "Quantification of liver viscoelasticity with acoustic radiation force: A study of hepatic fibrosis in a rat model," Ultrasound Med. Biol., vol. 39, no. 11, pp. 2091-2102, 2013.

[9] S. Chen, W. Sanchez, M. R. Callstrom, B. Gorman, J. T. Lewis, S. O. Sanderson, J. F. Greenleaf, H. Xie, Y. Shi, M. Pashley, V. Shamdasani, M. Lachman, and S. Metz, "Assessment of liver viscoelasticity by using shear waves induced by ultrasound radiation force," Radiology, vol. 266, no. 3, pp. 964-970, 2013.

[10] V. Rotemberg, M. Palmeri, R. Nightingale, N. Rouze, and K. Nightingale, "The impact of hepatic pressurization on liver shear wave speed estimates in constrained versus unconstrained conditions," Phys. Med. Biol., vol. 57, no. 2, p. 329-341, 2012.
[11] C. T. Barry, B. Mills, Z. Hah, R. A. Mooney, C. K. Ryan, D. J. Rubens, and K. J. Parker, "Shear wave dispersion measures liver steatosis," Ultrasound Med. Biol., vol. 38, no. 2, pp. 175-182, 2012.

[12] M. L. Palmeri, M. H. Wang, N. C. Rouze, M. F. Abdelmalek, C. D Guy, B. Moser, A. M. Diehl, and K. R. Nightingale, "Noninvasive evaluation of hepatic fibrosis using acoustic radiation force-based shear stiffness in patients with nonalcoholic fatty liver disease," $J$. Hepatol., vol. 55, no. 3, pp. 666-672, 2011.

[13] M. L. Palmeri, M. H. Wang, J. J. Dahl, K. D. Frinkley, and K. R. Nightingale, "Quantifying hepatic shear modulus in vivo using acoustic radiation force," Ultrasound Med. Biol., vol. 34, no. 4, pp. 546-558, 2008.

[14] J. Brum, M. Bernal, J. L. Gennisson, and M. Tanter, "In vivo evaluation of the elastic anisotropy of the human Achilles tendon using shear wave dispersion analysis," Phys. Med. Biol., vol. 59, no. 3, pp. 505-523, 2014.

[15] J.-L. Gennisson, N. Grenier, C. Combe, and M. Tanter, "Supersonic shear wave elastography of in vivo pig kidney: Influence of blood pressure, urinary pressure and tissue anisotropy," Ultrasound Med. Biol., vol. 38, no. 9, pp. 1559-1567, 2012.

[16] W. N. Lee, M. Pernot, M. Couade, E. Messas, P. Bruneval, A. Bel, A. A. Hagege, M. Fink, and M. Tanter, "Mapping myocardial fiber orientation using echocardiography-based shear wave imaging," IEEE Trans. Med. Imaging, vol. 31, no. 3, pp. 554-562, 2012.

[17] R. R. Bouchard, S. J. Hsu, M. L. Palmeri, N. C. Rouze, K. R. Nightingale, and G. E. Trahey, "Acoustic radiation force-driven assessment of myocardial elasticity using the displacement ratio rate (DRR) method," Ultrasound Med. Biol., vol. 37, no. 7, pp. 10871100, 2011.

[18] E. Mfoumou, J. Tripette, M. Blostein, and G. Cloutier, "Time-dependent hardening of blood clots quantitatively measured in vivo with shear-wave ultrasound imaging in a rabbit model of venous thrombosis," Thromb. Res., vol. 133, no. 2, pp. 265-271, 2014.

[19] K. Nightingale, S. McAleavey, and G. Trahey, "Shear-wave generation using acoustic radiation force: In vivo and ex vivo results," Ultrasound Med. Biol., vol. 29, no. 12, pp. 1715-1723, 2003.

[20] A. Hadj-Henni, C. Schmitt, and G. Cloutier, "Shear wave induced resonance elastography of soft heterogeneous media," J. Biomech., vol. 43, no. 8, pp. 1488-1493, 2010.

[21] C. Schmitt, A. Hadj-Henni, and G. Cloutier, "Ultrasound dynamic micro-elastography applied to the viscoelastic characterization of soft tissues and arterial walls," Ultrasound Med. Biol., vol. 36, no. 9, pp. 1492-1503, 2010.

[22] K. Nightingale, M. S. Soo, R. Nightingale, and G. Trahey, "Acoustic radiation force impulse imaging: In vivo demonstration of clinical feasibility," Ultrasound Med. Biol., vol. 28, no. 2, pp. 227-235, 2002.

[23] T. Deffieux, G. Montaldo, M. Tanter, and M. Fink, "Shear wave spectroscopy for in vivo quantification of human soft tissues viscoelasticity," IEEE Trans. Med. Imaging, vol. 28, no. 3, pp. 313-322, 2009.

[24] S. Callé, J.-P. Remenieras, O. B. Matar, M. E. Hachemi, and F. Patat, "Temporal analysis of tissue displacement induced by a transient ultrasound radiation force," J. Acoust. Soc. Am., vol. 118, no. 5, pp. 2829-2840, 2005.

[25] M. Elkateb Hachemi, S. Callé, and J. P. Remenieras, "Transient displacement induced in shear wave elastography: Comparison between analytical results and ultrasound measurements," Ultrasonics, vol. 44, suppl. 1, pp. e221-e225, 2006.

[26] O. V. Rudenko, A. P. Sarvazyan, and S. Y. Emelianov, "Acoustic radiation force and streaming induced by focused nonlinear ultrasound in a dissipative medium," J. Acoust. Soc. Am., vol. 99, no. 5, pp. 2791-2798, 1996.

[27] D. Ekeom, A. Hadj-Henni, and G. Cloutier, "Design of a phased array for the generation of adaptive radiation force along a path surrounding a breast lesion for dynamic ultrasound elastography imaging," IEEE Trans. Ultrason. Ferroelectr. Freq. Control, vol. 60, no. 3 , pp. $552-561,2013$.

[28] P. Acevedo and D. Das-Gupta, "The measurement of the spatial average temporal average intensity $I_{\text {sata }}$ and ultrasonic power $W$ in composite ultrasonic transducers for medical application," Ultrasonics, vol. 40, no. 1-8, pp. 819-821, 2002.

[29] M. E. Stratmeyer and F. L. Lizzi, "Special issue on the biological effects of ultrasound," IEEE Trans. Ultrason. Ferroelectr. Freq. Control, vol. 33, no. 2, pp. 137-138, 1986.

[30] J. L. Gennisson and G. Cloutier, "Sol-gel transition in agar-gelatin mixtures studied with transient elastography," IEEE Trans. Ultrason. Ferroelectr. Freq. Control, vol. 53, no. 4, pp. 716-723, 2006. 
[31] J. B. Robert, Underwater Electroacoustic Measurements, Washington, DC, USA: Naval Research Laboratory, 1970.

[32] P. A. Narayana and J. Ophir, "A closed form method for the measurement of attenuation in nonlinearly dispersive media," Ultrason. Imaging, vol. 5, no. 1, pp. 17-21, 1983.

[33] T. A. Bigelow and Y. Labyed, "Chapter 4: Attenuation compensation and estimation," in Quantitative Ultrasound in Soft Tissues, J. Mamou and M. L. Oelze, Eds., Dordrecht, The Netherlands: Springer, 2013, pp. 71-93.

[34] P. A. Narayana and J. Ophir, "On the frequency dependence of attenuation in normal and fatty liver," IEEE Trans. Sonics Ultrason. vol. 30, no. 6, pp. 379-382, 1983.

[35] M. Tozaki, M. Saito, C. Joo, M. Yamaguchi, S. Isobe, Y. Ogawa, K. Homma, and E. Fukuma, "Ultrasonographic tissue quantification of the breast using acoustic radiation force impulse technology: Phantom study and clinical application," Jpn. J. Radiol., vol. 29, no. 8, pp. 598-603, 2011.

[36] E. Montagnon, A. Hadj-Henni, C. Schmitt, and G. Cloutier, "Rheological assessment of a polymeric spherical structure using a threedimensional shear wave scattering model in dynamic spectroscopy elastography," IEEE Trans. Ultrason. Ferroelectr. Freq. Control, vol. 61, no. 2, pp. 277-287, 2014.

[37] M. O. Culjat, D. Goldenberg, P. Tewari, and R. S. Singh, "A review of tissue substitutes for ultrasound imaging," Ultrasound Med. Biol., vol. 36, no. 6, pp. 861-873, 2010.

[38] S. B. Barnett, G. R. Ter Haar, M. C. Ziskin, H.-D. Rott, F. A. Duck, and K. Maeda, "International recommendations and guidelines for the safe use of diagnostic ultrasound in medicine," Ultrasound Med. Biol., vol. 26, no. 3, pp. 355-366, 2000

[39] H. Zhao, P. Song, M. W. Urban, J. F. Greenleaf, and S. Chen, "Shear wave speed measurement using an unfocused ultrasound beam," $\mathrm{Ul}$ trasound Med. Biol., vol. 38, no. 9, pp. 1646-1655, 2012.

[40] S. Vaezy, R. Martin, and L. Crum, "Therapeutic ultrasound, part II, high intensity focused ultrasound: A method of hemostasis," Echocardiography, vol. 18 , no. 4 , pp. 309-315, 2001.

[41] K. J. Taylor, C. A. Riely, L. Hammers, S. Flax, G. Weltin, G. Garcia-Tsao, H. O. Conn, R. Kuc, and K. W. Barwick, "Quantitative US attenuation in normal liver and in patients with diffuse liver disease: Importance of fat," Radiology, vol. 160, no. 1, pp. 65-71, 1986.

[42] Y. Fujii, N. Taniguchi, K. Itoh, K. Shigeta, Y. Wang, J.-W. Tsao, K. Kumasaki, and T. Itoh, "A new method for attenuation coefficient measurement in the liver: Comparison with the spectral shift central frequency method," J. Ultrasound Med., vol. 21, no. 7, pp. 783-788, 2002 .

[43] M. Tozaki, S. Isobe, and E. Fukuma, "Preliminary study of ultrasonographic tissue quantification of the breast using the acoustic radiation force impulse (ARFI) technology," Eur. J. Radiol., vol. 80 no. 2, pp. e182-e187, 2011

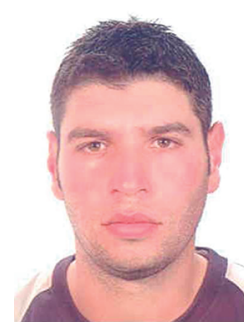

Abderrahmane Ouared completed his undergraduate studies at the Université des sciences de Montpellier in France. He received his master's degree in medical imaging and physics from the Université François-Rabelais de Tours, France, in 2009. He is doing his Ph.D. degree in biomedical engineering at the University of Montreal in Canada, where he is conducting his research in the Laboratory of Biorheology and Medical Ultrasonics at the University of Montreal Hospital Research Center. His research interests include medical ultrasound imaging, dynamic elastography and applications in breast cancer, acoustic radiation force generation, and shear wave tracking.

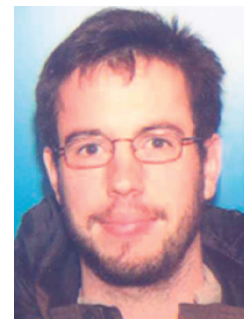

Emmanuel Montagnon was born in 1982 in Nancy, France. He obtained his B.Sc. degree in theoretical physics from the Université de Nantes and his master's degree from the Université de Grenoble in 2007. He completed his Ph.D. degree in biomedical engineering at the University of Montreal in Canada, where he was conducting his research in the Laboratory of Biorheology and Medical Ultrasonics at the University of Montreal Hospital Research Center. His research interests included dynamic ultrasound elastography and applications to breast cancer and deep vein thrombosis, shear wave scattering modeling, and plane wave imaging.

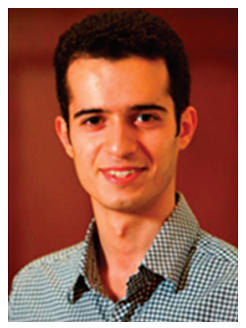

Siavash Kazemirad obtained his B.Sc. degree in mechanical engineering from the Iran University of Science and Technology in 2006. He obtained his M.Sc. degree in mechanical engineering from the Sharif University of Technology in 2009, majoring in biomechanics. He completed his Ph.D. degree in mechanical engineering in 2013 at McGill University, Montreal, Canada, where he worked at the Biomechanics Research Laboratory. Upon completing his Ph.D. degree in 2013, he joined the Laboratory of Biorheology and Medical Ultrasonics at the University of Montreal Hospital Research Centre as a postdoctoral fellow. His research interests include vibration and acoustics, wave propagation, ultrasound imaging, material characterization, and dynamic ultrasound elastography.

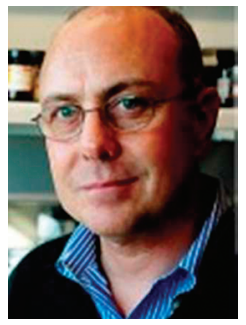

Louis Gaboury graduated from medical school (Université de Montréal) in 1978, completed a residency program in anatomic-pathology in 1983, and obtained a Ph.D. degree in experimental pathology from the University of British Columbia (1988). His research and clinical interests concern breast cancer and molecular pathology. He is an attending pathologist at the Centre hospitalier de l'Université de Montréal (University of Montreal Hospital), Full Professor of pathology, and currently Chairman of the Department of Pathology and Cellular Biology at the Université de Montréal.

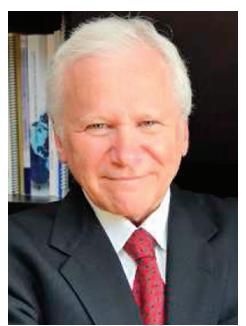

André Robidoux is currently Professor of Surgery (tenure) and Scotia Chair in diagnosis and treatment of breast cancer at the University of Montreal. Dr. Robidoux is a graduate of the University of Montreal where he received both the baccalaureate of arts and doctor of medicine degrees. He completed his postgraduate training in surgery in Montreal and in the Department of Developmental Therapeutics at MD Anderson Cancer Centre, University of Texas in Houston. He was responsible for the creation of the Eli Lilly Breast Cancer Research Fund at the University of Montreal and contributed to the creation of the CIBC Chair in Basic Research in Breast Cancer at the same institution. In 2002, he received the University of Montreal rector award for his exceptional contribution to the development of the Faculty of Medicine. He carried out more than 40 clinical trials to test the concept of neo adjuvant chemotherapy in palpable operable breast cancer and evaluate the work of different therapeutic strategies. He is the author of more than 75 peer-reviewed publications and has been invited speaker to 319 conferences.

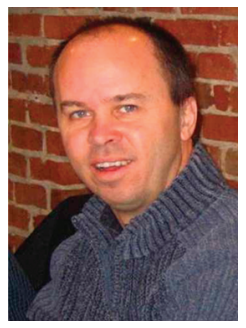

Guy Cloutier (S'89-M'90-SM'07) obtained his B.Eng. in electrical engineering (1984), and M.Sc and Ph.D. in biomedical engineering $(1986,1990)$ Between 1990 and 1992, he was a postdoctoral fellow at The Pennsylvania State University with Prof. K. Kirk Shung. Dr. Cloutier is Director of the Laboratory of Biorheology and Medical Ultrasonics at the University of Montreal Hospital Research Center (www.lbum-crchum.com), Professor and Director of Research in the Department of Radiology, Radio-oncology and Nuclear Medicine, and Member of the Institute of Biomedical Engineering at the University of Montreal. His research interests are in quantitative ultrasound imaging of red blood cell aggregation; quasi-static and dynamic ultrasound elastography of atherosclerotic plaques, vascular aneurysms, deep vein 
thrombi, breast cancers and liver steatosis; 3-D morphologic and hemodynamic assessment of lower limb arterial stenoses; development of multi-physics imaging methods; and mathematical and biomechanical modeling. He has published more than 160 peer-reviewed articles in these fields, holds 12 patents, licensed two technologies, and was recipient of the National Scientist award of the Fonds de la Recherche en
Santé du Québec (2004-2009). Dr. Cloutier is Associate Editor of IEEE Transactions on Ultrasonics, Ferroelectric and Frequency Control, Invited Associate Editor of Medical Physics, and member of the international advisory editorial board of Current Medical Imaging Reviews. He was a member of the advisory editorial board of Ultrasound in Medicine and Biology from 1999 to 2014. 